\title{
Primary lateral sclerosis: clinical, neurophysiological, and magnetic resonance findings
}

\author{
J Kuipers-Upmeijer, A E J de Jager, J M Hew, J W Snoek, T W van Weerden
}

\begin{abstract}
Objective-To describe the clinical, neurophysiological, and MRI findings in 10 patients with primary lateral sclerosis (PLS).

Results-The course of the disease was very slowly progressive. Spasticity due to upper motor neuron dysfunction was the most prominent sign, but EMG showed slight lower motor neuron signs, such as a mixed pattern on maximal voluntary contraction and enlarged motor unit potentials. One patient had clinically mild lower motor neuron involvement. Central motor conduction times (CMCT) were more prolonged in PLS than is the case in ALS. Minor sensory signs were found on neurophysiological examination, comparable with those in ALS. In four patients serum creatine kinase activity was raised. On MRI cortical atrophy was seen, most pronounced in the precentral gyrus and expanding into the parietal-occipital region.

Conclusions-PLS is a distinct clinical syndrome, part of the range of motor neuron diseases. Besides pronounced upper motor neuron symptoms, mild lower motor neuron symptoms can also be found, as well as (subclinical) sensory symptoms. PLS can be distinguished from ALS by its slow clinical course, a severely prolonged MEP, and a more extensive focal cortical atrophy.

(f Neurol Neurosurg Psychiatry 2001;71:615-620)
\end{abstract}

Keywords: primary lateral sclerosis; motor neuron disease

Primary lateral sclerosis (PLS) is a disorder characterised by spinobulbar spasticity due to upper motor neuron degeneration. It has been a much debated entity in the past, because many reported patients seemed ultimately to have different diseases-for example, amyotrophic lateral sclerosis (ALS), spinal multiple sclerosis, or cervical spondylotic myelopathy. Younger et al revived the syndrome on the basis of pathological observations and modern technical findings. ${ }^{1}$ In 1992 Pringle et al reported on a group of eight patients with PLS and established clinical and laboratory criteria. ${ }^{2}$ According to modern views, PLS is considered to be part of the range of motor neuron diseases consisting of primary lateral sclerosis, amyotrophic lateral sclerosis (ALS), and progressive spinal muscular atrophy (PSMA). ${ }^{3-6}$
Primary lateral sclerosis stands here for a dysfunction of the corticospinal pathway without lower motor neuron involvement. Clinical features include prominent spasticity with only slight weakness in the lower limbs and eventually pseudobulbar symptoms (dysarthria and compulsive laughing or crying). The disease is slowly progressive. Although "pure" PLS remains confined to the spinobulbar tract and spares the lower motor neuron, transitions to ALS have been found. ${ }^{6}$ Pathologically, a selective involvement of the motor cortex is seen with degeneration of the Betz cells and demyelination of the descending motor tracts. ${ }^{28}$ Brain MRI has shown atrophy of the precentral gyrus in many cases. ${ }^{6}{ }^{910}$ We present here the clinical and neurophysiological findings in 10 patients with PLS and describe the abnormalities on MRI.

\section{Materials and methods}

Over a period of 15 years, 10 patients with PLS were seen in the departments of neurology of the University Hospital and the Martini Hospital in Groningen. Diagnosis was in accordance with the criteria set by Pringle et $a l^{2}$ Nine patients were personally examined by one of us (J K-U); one patient had died, but his medical records could be traced. An extensive serological screening and CSF examination was carried out in all patients.

Motor nerve conduction velocities (MNCVs) were measured according to standard procedures (median nerve normal $50 \mathrm{~m} / \mathrm{s}$ and peroneal nerve $42 \mathrm{~m} / \mathrm{s}$ ). Sural nerve conduction velocity (SNCV) and response amplitudes were studied with the retrograde method (normal conduction velocity 40-57 $\mathrm{m} / \mathrm{s}$, amplitude 5-20 $\mu \mathrm{V}$ ). Concentric needle EMG was performed in the tibialis anterior muscle, extensor digitorum communis muscle, and the biceps brachii muscle on the left side.

In eight patients transcranial magnetic stimulation was performed with a Magstim model 200, coil diameter $90 \mathrm{~mm}$, output $80 \%$ $100 \%$. Recording was done with surface electrodes for the arm from the abductor pollicis brevis and for the leg from the tibial anterior muscle. All measurements were taken during slight voluntary activation of these muscles. The central motor conduction time (CMCT) was calculated by subtracting the latencies at cervical (C6/C7) or lower lumbar (L4/L5) stimulation from the latencies at cortical stimulation. Normal mean values (SD) of the CMCT during slight activation of the stimulated muscle are for the abductor pollicis brevis 


\begin{tabular}{|c|c|c|c|c|c|c|c|c|c|c|}
\hline & \multicolumn{10}{|l|}{ Patient } \\
\hline & 1 & 2 & 3 & 4 & 5 & 6 & 7 & 8 & 9 & 10 \\
\hline Sex & M & $M$ & M & $M$ & $M$ & $M$ & $M$ & $M$ & M & $\mathrm{V}$ \\
\hline Age of onset (y) & 54 & 45 & 44 & 39 & 31 & 23 & 55 & 30 & 57 & 53 \\
\hline Family history & - & - & - & - & - & - & - & - & - & - \\
\hline Duration in years & 6 & 8 & 18 & 7 & 35 & 10 & 6 & 11 & 8 & 18 \\
\hline First diagnosis & ALS & $\mathrm{CM}$ & LS & MS & ALS & LS & Park & MS & ALS & LS \\
\hline Diagnostic delay (y) & 5 & 2 & 0 & 6 & 28 & 0 & 2 & 10 & 3 & 0 \\
\hline Weakness & - & - & - & - & + & - & + & - & - & - \\
\hline Site of onset & L leg & L leg & Legs & Legs & $\mathrm{R}$ arm & Legs & $\mathrm{R}$ leg & Legs & Speech & L leg \\
\hline Spasticity/Ashworth & 3 & 2 & 3 & 2 & 2 & 2 & NT & 2 & 2 & 2 \\
\hline LMN signs & - & - & - & - & - & - & - & - & - & + \\
\hline Sensory deficit & - & - & - & - & - & - & - & - & - & - \\
\hline Pseudobulbar affect & + & + & - & - & - & - & + & - & + & + \\
\hline Urinary incontinence & + & + & - & - & - & - & - & - & - & + \\
\hline Barthel/Rankin scale & $16 / 3$ & $16 / 3$ & $18 / 2$ & $20 / 2$ & $4 / 5$ & $20 / 2$ & $11 / 4$ & $18 / 2$ & $17 / 3$ & $17 / 3$ \\
\hline Medical aids & $\mathrm{WCH}$ & WCH & $\mathrm{W}$ & - & $\mathrm{WCH}$ & - & $\mathrm{WCH}$ & W & $\mathrm{W}$ & $\mathrm{WCH}$ \\
\hline
\end{tabular}

(5.6 ms (SD 0.9) $\mathrm{ms})$ and for the tibial anterior muscle $((9.5 \times \mathrm{L}-1.7) \mathrm{ms}(\mathrm{SD} 1.64) \mathrm{ms}$; $\mathrm{L}$ is body length $(\mathrm{m}))$. Short latency somatosensory evoked responses were determined as described by Chiappa. ${ }^{11}$ The means (SD) were used as reference values.

In nine patients a cranial MRI was obtained with a Siemens 1.5 Tesla scanner (Magneton Vision). Sagittal T1 weighted (TR/TE 430/12), coronal T2 weighted (TSE TR/TE 3950/99), axial T2 weighted (TSE TR/TE 4000/90), and axial dark fluid (TR/TE 9999/105) images were obtained for seven patients. The MRI of two patients some years previously were re-evaluated. A cervical MRI was obtained for six patients and a myelography for one.

In all patients the degree of disability was scored using the Barthel index and the modified Rankin scale. ${ }^{12}$

Non-parametric tests (Spearman's $r$, MannWhitney $U, \chi^{2}$ ) were used to assess the relations of the following variables: age, age of onset, disease duration, degree of spasticity, presence of pseudobulbar signs, urinary incontinence, serum creatine kinase concentration, CSF protein concentration, and abnormal motor unit potentials on EMG. Because there were no directional hypotheses, two sided tests were used to determine the level of significance $(\mathrm{p}<0.05)$.

\section{Results}

The group consisted of nine men and one woman. Their clinical features are depicted in table 1. The main finding in all patients was severe spasticity with accompanying corticospinal tract signs such as hyperreflexia, Babinski's sign, pseudobulbar reflexes, and masseter hyperreflexia. The symptoms started in eight patients in the legs and were asymmetric in five patients. Spasticity was scored according to the modified Ashworth scale: grade 0 (no increase in tone), grade 1 (slight increase in tone, giving a "catch" when the affected part is moved), grade 2 (more pronounced increase in tone, but affected part easily flexed), grade 3 (considerable increase in tone; passive movement difficult), grade 4 (affected part rigid in flexion or extension). ${ }^{13}$ One patient had atrophy of the first interosseus, abductor digiti minimi, and abductor pollucis muscles in both hands, with normal strength and sensation and without fasciculations. The atrophy had developed very gradually over more than 8 years. An extensive search showed no cause for this abnormality. None of the other patients had clinical signs of lower motor neuron involvement.

Peripheral blood cell count was normal in all patients. Serum values for hepatic and renal function tests, calcium, and phosphate were normal. Creatine kinase was slightly increased in four out of nine patients. Immunoelectrophoresis was normal in nine patients and paraproteinaemia was absent. Anti-GM1 antibodies, hexosaminidase concentrations, very long chain fatty acid concentrations, vitamin B12 concentrations, and HTLV-1 antibodies were negative or normal in nine patients and not measured in patient 7 . Normal serology or normal CSF in all patients excluded Lues and Borrelia infections. HIV serology was not performed because no patient had risk factors

Table 2 Laboratory findings

\begin{tabular}{|c|c|c|c|c|c|c|c|c|c|c|}
\hline & \multicolumn{10}{|l|}{ Patient } \\
\hline & 1 & 2 & 3 & 4 & 5 & 6 & 7 & 8 & 9 & 10 \\
\hline Serum chemistry & Norm & Norm & Norm & Norm & Norm & Norm & Norm & Norm & Norm & Norm \\
\hline Creatine kinase & 62 & 25 & 20 & 66 & 68 & 23 & 22 & 24 & 90 & NT \\
\hline Infectious serologies & Norm & Norm & Norm & Norm & Norm & Norm & Norm & Norm & Norm & Norm \\
\hline Immune-electrophoresis & Norm & Norm & Norm & Norm & Norm & Norm & NT & Norm & Norm & Norm \\
\hline Paraproteinaemia & - & - & - & - & - & - & NT & - & - & - \\
\hline Anti GM1 antibodies & - & - & - & - & - & - & NT & - & - & NT \\
\hline \multicolumn{11}{|l|}{ CSF: } \\
\hline Protein & 0.39 & 0.67 & 0.48 & 0.40 & 0.32 & 0.53 & 0.77 & 0.42 & 0.74 & 0.43 \\
\hline $\mathrm{OB} / \mathrm{IgG}$ index & Norm & Norm & Norm & Norm & Norm & Norm & Norm & Norm & Norm & Norm \\
\hline Cell count & Norm & Norm & Norm & Norm & Norm & Norm & Norm & Norm & Norm & Norm \\
\hline
\end{tabular}

Norm=Normal; -=absent; NT=not tested; $\mathrm{OB}=$ oligoclonal bands; $\mathrm{CK}$ normal $=<0-50 \mathrm{u} / \mathrm{l}$; CSF protein normal=<0.55 g/l. 


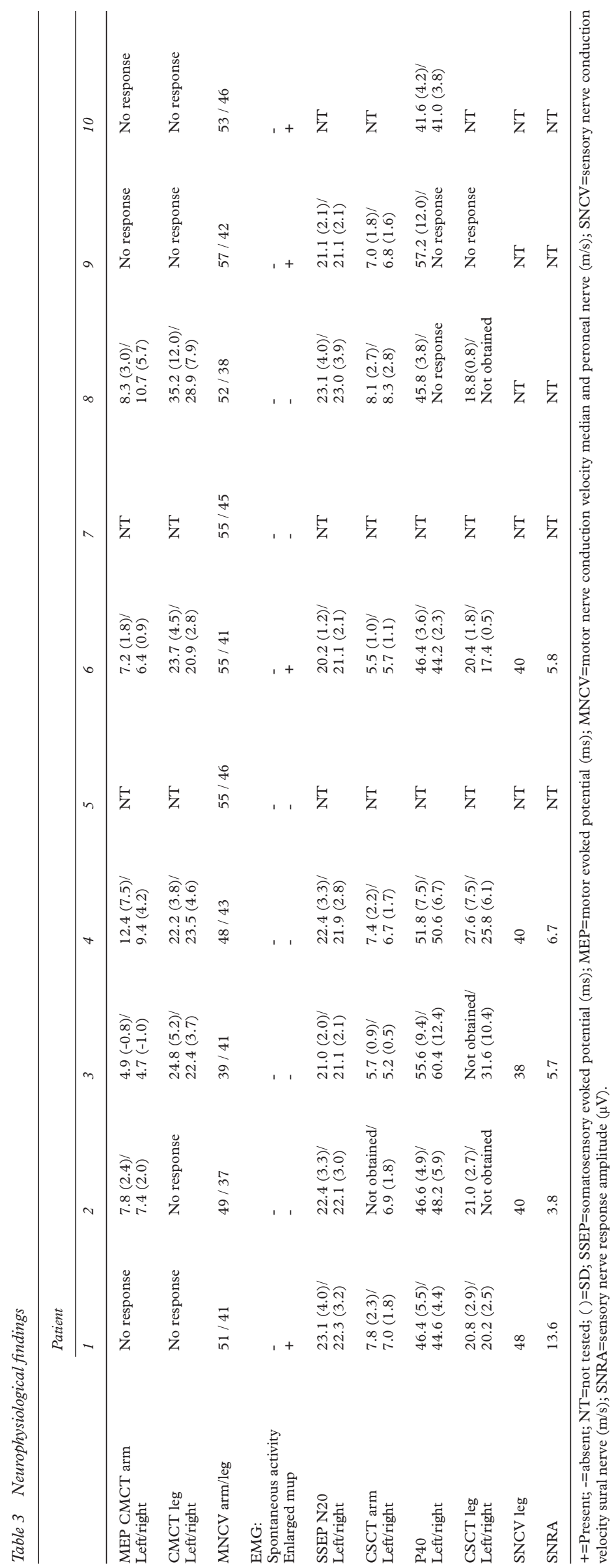

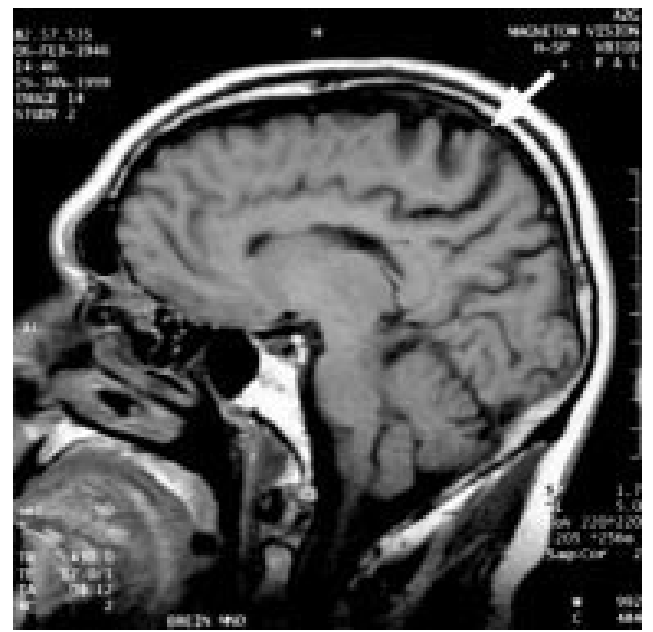

Figure 1 MRI. Sagittal T1 weighted image showing atrophy of the precentral gyrus (arrow).

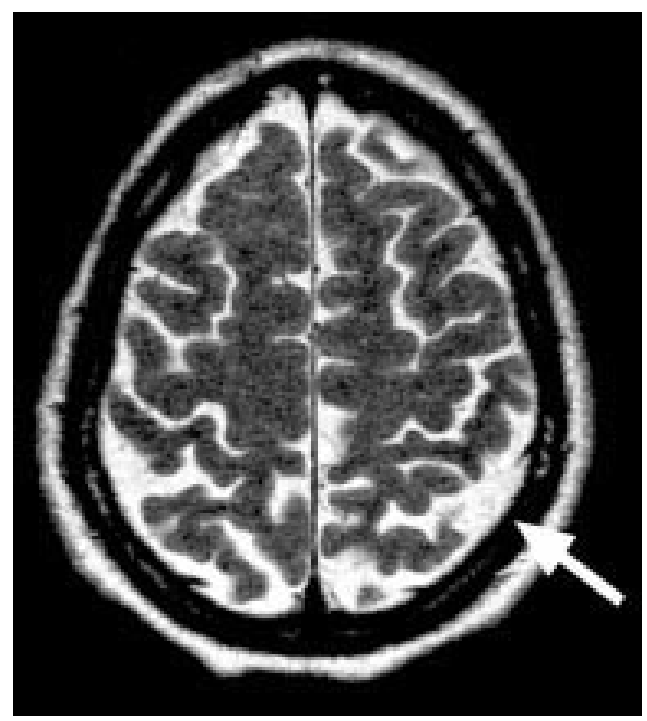

Figure 2 Axial T2 weighted image showing parietal-occipital arophy (arrow).

for HIV. The CSF was normal with the exception of a slightly increased protein concentration in three patients (table 2).

The results of the motor nerve conduction velocity (MNCV), needle EMG, motor evoked potential (MEP), and somatosensory evoked potential (SSEP) examinations are presented in table 3.

Cranial MRI showed not only conspicuous precentral atrophy but also atrophy in the frontal and parietal-occipital regions (fig 1 and 2). Cervical MRI or myelography was normal for all patients tested.

In the statistical analysis only a few significant results were found. Age of onset was significantly later for patients with pseudobulbar signs (Mann-Whitney $\mathrm{p}<0.01$ ). In our series pseudobulbar signs were only present when the onset of the disease occurred at the age of 45 or later. No significant differences in chronological age or duration of the disease were found between these two subgroups. The absence of pseudobulbar signs was associated with the absence of urinary incontinence $\left(\chi^{2}=4.29, \mathrm{p}<0.040\right)$. 


\section{Discussion}

The diagnosis PLS is made on clinical grounds. Commonly used criteria are adult onset, negative family history, gradual progression, spasticity with paresis of the legs for more than 3 years, and dysfunction clinically limited to the corticospinal tracts. ${ }^{2}$ Differential diagnosis of PLS includes many spinal cord syndromes. In all our patients the normal CSF or the absence of typical white matter densities on MRI ruled out chronic spinal MS. Infections such as HTLV-1, neuroborreliosis, or neurosyphilis were excluded by the normal serology or the normal CSF. Subacute combined degeneration of the cord due to vitamin B12 deficiency and metabolic diseases (adrenoleucodystrophy, hexosaminidase deficiency, cerebrotendinous xanthomatosis) were excluded in nine patients. Foramen magnum lesions or compression of the cord was excluded on clinical grounds and confirmed by cervical MRI or myelography in seven patients. Hereditary spastic paralysis was ruled out by a meticulous family history. PLS may be associated with malignancies but in our group these were absent. $^{14} 15$

PLS usually is equally distributed between both sexes. ${ }^{2}$ In our series, however, there was a male preponderance. Median age of onset was 44.5 years. In all patients the clinical course was slowly progressive, from 6 to 35 years. There was no correlation between the duration of the disease and any of the clinical, laboratory, or EMG data. Three patients were diagnosed as PLS, but seven were at first misdiagnosed: three as ALS, two as multiple sclerosis, one as Parkinson's disease, and one as cervical compression myelopathy. It took between 2 and 28 years before the correct diagnosis was made. All patients had difficulties in walking, but only two patients had manifest weakness in the legs. In all cases the stiff, awkward, wooden way of walking was due to extreme spasticity. According to the Ashworth scale, seven patients were in grade 2 , and two in grade 3 . No correlation was found between the Ashworth scale and any of the other data. Five patients had bouts of unprovoked laughing and crying. This group with pseudobulbar signs differed significantly from the five others by their older age of onset. The patients without pseudobulbar signs had no urinary incontinence. One patient had clinical signs of lower motor neuron involvementnamely, an atrophy of the muscles of both hands, without weakness, fasciculations, or sensory loss. This atrophy had been present for more than 8 years and was very slowly progressive. No atrophy was present in other parts of the body. In the literature a few similar patients have been described. ${ }^{7}$ Three of our patients had urinary incontinence, probably due to bladder hyperreflexia. ${ }^{26}$ On detailed neuropsychological evaluation, mild cognitive impairment has been found in PLS, especially in frontal lobe function and memory. ${ }^{16}$ Our patients had no clinical signs of intellectual deterioration.

The Barthel index (BI) is a disability score; patients with a score of less than 17 are unable to live independently. The modified Rankin scale (MRS) measures handicap; a score of 2 or more means dependency. Our patients with a $\mathrm{BI} / \mathrm{MRS}=20 / 2$ could walk; the group of about $18 / 2$ had a rollator, the others a wheelchair. Two patients were severely handicapped; these were the only ones with manifest loss of strength. Some patients had a remarkably good score even after many years of illness. This is by contrast with patients with ALS where the increase in dependency is measured in months. ${ }^{17} 18$

Serum creatine kinase may be increased in patients with ALS or PSMA, but no increase has ever been reported in patients with PLS. ${ }^{19}$ It was slightly increased in four of nine of our patients. An increased creatine kinase did not correlate with any of the clinical or EMG data. Protein concentrations in CSF are increased in $20 \%-30 \%$ of patients with ALS. $^{2021}$ Little is known about the CSF in patients with PLS, although an increased protein concentration has been mentioned. ${ }^{169}$ Three of our patients had an increased CSF protein.

Cortically evoked motor potentials are often absent in patients with PLS. When present, the central motor conduction time (CMCT) may be considerably prolonged, two to three times normal, by contrast with ALS where CMCT is normal or only slightly delayed. ${ }^{22-24}$ In three of our patients MEPs could not be elicited in the arms and legs, and in one only in the arms. Two patients had a normal CMCT to the arms $(<2$ $\mathrm{SD}$ ); in the others CMCT was prolonged in arms and legs. In the legs this prolongation varied from 2.9 to $12.0 \mathrm{SD}$ (mean 5.6 SD). The difference in CMCT between PLS and ALS is not easy to explain. Both disorders are due to the death of large pyramidal neurons in the cortex. In PLS the very protracted course (compared with ALS) may ultimately cause secondary demyelination of the pyramidal tracts, thus leading to a prolonging of the CMCT. We found considerable asymmetry for the CMCT in some patients; this has been mentioned before. ${ }^{24}$ The lower motor neuron remains spared in PLS, although occasionally slight signs of denervation in the EMG are found. ${ }^{1622}$ None of our patients had acute denervation potentials but in four patients enlarged motor unit potentials (MUPs) and a mixed pattern on maximal voluntary contraction were present. These may be considered as signs of chronic denervation caused by slow lower motor neuron loss. There was no correlation between the presence of enlarged mups and the clinical or laboratory data of the patients.

Motor nerve conduction velocity in PLS is normal or prolonged. ${ }^{622} 25$ More than half of the patients in our group had slightly prolonged conduction velocities or values near the lower limit of normal in both upper and lower limbs. Slowing of MNCV has been found in patients with ALS where a preferential degeneration of the fast conducting myelinated axons in the peripheral nerve is presumed. ${ }^{26}$ The MNCV findings in our patients are comparable with those found in ALS. 
Somatosensory evoked potentials in patients with PLS are reported to be normal or prolonged..$^{22}{ }^{28}$ We found at stimulation of the median nerve in three patients a prolonged central sensory conduction time (CSCT) $(>2$ SD). No responses could be obtained of the peroneal nerve in one patient. In the others CSCT was normal (two patients) or prolonged (from 2.5 to $10.4 \mathrm{SD}$, mean 5.4 SD). Despite this, the sensory system was clinically normal in all patients. ${ }^{27}$ In three patients no values were obtained in the arms or legs because of a lack of reproducible cervical or lumbar responses. In five patients the peripheral sensory system was tested. Sensory nerve response amplitude (SNRA) was diminished in one patient but normal in the others. Sensory nerve conduction velocity was near the lower limit of normal in all patients. On pathological examination the sensory system is normal in PLS, especially in the posterior columns that are thought to convey the SSEP impulses. ${ }^{8}$ In patients with ALS a disturbed SSEP can also be found. Here a cortical origin has been suggested, ${ }^{29}$ as well as a combined involvement of peripheral and central pathways. ${ }^{26}$ Our findings in PLS match this second explanation.

In patients with PLS MRI shows atrophy of the frontoparietal part of the brain, most evident in the precentral area, with concomitant degeneration of the underlying white matter. ${ }^{2610}$ This can be seen most clearly on images in the parasagittal plane. The mean surface area in this region may be reduced to $75 \%$ of normal. ${ }^{10}$ In early stages of the disease this is probably not as prominent as later on. ${ }^{2}{ }^{9}$ These findings are by contrast with ALS where the size of the precentral gyrus is not different from controls, although shrinkage of the white matter is sometimes present. ${ }^{10}$ This difference in atrophy between PLS and ALS may be explained by the long duration of the disease in PLS. Brain MRI was carried out in nine of our patients. All showed atrophy of the frontal region, most prominent in the precentral gyrus (fig 1). We also saw atrophy in the parietaloccipital region of the brain (fig 2). Atrophy was most clearly depicted in the parasagittal images. Seven patients had a moderate enlargement of the ventricles. We considered this as secondary to the brain atrophy. In PLS as well as in ALS, increased signal intensities have been described at the site of the pyramidal tract on T2 weighted images. ${ }^{20}$ In only one patient did we see increased intensity in the posterior limb of the internal capsule extending into the corona radiata.

We conclude that upper motor neuron signs predominate clinically in patients with PLS, although, in rare cases, slight lower motor neuron symptoms may be present. On neurophysiological examination, however, lower motor neuron signs can be found more often.Primary lateral sclerosis has therefore to be considered as a part of the range of motor neuron diseases with predominant, but not exclusive, upper motor neuron involvement. We also found slight deficits in the sensory system, resembling those seen in ALS. Primary lateral sclerosis can be distinguished from ALS by the long duration of the disease, the extensive cortical atrophy and the considerable prolonging of the MEP. This distinction is important for the patient because the diagnosis of PLS does not have the same life threatening implications as ALS, at least not in the short term. The fewer lower motor neuron signs, the better the prognosis.

1 Younger DS, Chou S, Hays AP, et al. Primary lateral sclerois. A clinical diagnosis re-emerges. Arch Neurol 1988;45:1304-7.

2 Pringle CE, Hudson AJ, Munoz DG, et al. Primary lateral sclerosis. Clinical features, neuropathology and diagnostic criteria. Brain 1992;115:495-520.

3 Donaghy $M$. Classification and clinical features of motor neuron diseases and motor neuropathies in adults. $\mathcal{F}$ Neurol 1999;246:331-3.

4 Norris F, Shepherd R, Denys E, et al. Onset, natural history and outcome in idiopathic adult motor neuron disease. $f$ Neurol Sci 1993;118:48-55

5 Swash M, Desai J, Misra VP. What is primary lateral sclerosis? F Neurol Sci 1999;170:5-10.

6 Le Forestier N, Maisonobe T, Spelle L, et al. Primary lateral sclerosis: further clarification. I Neurol Sci 2001;185: 95-100

7 Bruyn RPM, Koelman JHTM, Troost D, et al. Motor neuron disease (amyotrophic lateral sclerosis) arising from longstanding primary lateral sclerosis. $\mathcal{F}$ Neurol Neurosurg Psychiatry 1995;58:742-4.

8 Hudson AJ, Kiernan JA, Munoz DG, et al. Clinicopathological features of primary lateral sclerosis are different from amyotrophic lateral sclerosis. Brain Res Bull 1993;30: 59-64.

9 Heene R, Kolander D, Knisatschek H. Die chronischprogrediente spinobulbäre Spastik (primäre Lateralsklerose). Fortschr Neurol Psychiatr 1996;64:192-203.

10 Kiernan JA, Hudson AJ. Frontal lobe atrophy in motor neuron diseases. Brain 1994;117:747-57.

11 Chiappa KH. Short-latency somatosensory evoked potentials. Methodology. In: Chiappa KH, ed. Evoked potentials in clinical medicine. 3rd ed. Philadelphia: Lippincott-Raven, 1997:283-339.

12 Sulter G, Steen C, Keyser J de. Use of the Barthel index and modified Rankin scale in acute stroke trials. Stroke 1999;30:1538-41.

13 Middel B, Kuipers-Upmeijer H, Bouma J, et al. Effect of intrathecal baclofen delivered by an implanted programmable pump on health related quality of life in patients with severe spasticity. F Neurol Neurosurg Psychiatry 1997;63: $204-9$.

14 Desai J, Swash M. IgM paraproteinemia in a patient with primary lateral sclerosis. Neuromuscul Disord 1999;9:38-40.

5 Rowland LP Paraneoplastic primary lateral sclerosis and amyotrophic lateral sclerosis. Ann Neurol 1997;41:703-30.

Caselli RJ, Smith BE, Osborne D. Primary lateral sclerosis: a neuropsychological study. Neurology 1995;45:2005-9.

17 Meucci N, Nobile-Orazio E, Scarlato G. Intravenous immunoglobulin therapy in amyotrophic lateral sclerosis. $\mathcal{F}$ Neurol 1996;243:117-20

18 Pinto AC, Evangelista T, Carvalho M, et al. Respiratory assistance with a non-invasive ventilator (Bipap) in MND/ ALS patients: survival rates in a controlled trial. $\mathcal{F}$ Neurol Sci 1995;129(suppl): 19-26.

19 Sinaki M, Mulder DW. Amyotrophic lateral sclerosis: relationship between serum creatine kinase level and patient survival. Arch Phys Med Rehabil 1986;67:169-71.

20 Westarp ME, Schreiber H, Westarp MP, et al. Cerebral magnetic resonance imaging pathology and cerebro-spinal fluid protein in sporadic amyotrophic lateral sclerosis (sALS). Clin Neuropathol 1995;14:228-32.

21 Younger DS, Rowland LP, Latov N, et al. Motor neuron disease and amyotrophic lateral sclerosis: relation of high CSF protein content to paraproteinemia and clinical syndromes. protein content to parapro

22 Brown WF, Ebers GC, Hudson AJ, et al. Motor-evoked responses in primary lateral sclerosis. Muscle Nerve 1992;15:626-9.

23 Caramia MD, Cicinelli P, Paradiso C, et al. Excitability changes of muscular responses to magnetic brain stimulation in patients with central motor disorders. Electroencephalogr Clin Neurophysiol 1991;81:243-50.

24 Salerno A, Carlander B, Camu W, et al. Motor evoked potentials (MEPs): evaluation of the different types of responses in amyotrophic lateral sclerosis and primary lateral sclerosis. Electromyogr Clin Neurophysiol 1996;36: 361-8.

25 Russo LS Jr. Clinical and electrophysiological studies in primary lateral sclerosis. Arch Neurol 1982;39:662-4.

26 Theys PAI, Peeters E, Robberecht W. Evolution of motor and sensory deficits in amyotrophic lateral sclerosis and sensory deficits in amyotrophic lateral sclerosis 246:438-42. 
27 Aalfs CM, Koelman JHTM, Aramideh M, et al. Posterior tibial nerve somatosensory evoked potentials in slowly proparative study with clinical signs. $\mathcal{F}$ Neurol 1993;240:351-6.

28 Georgesco M, Salerno A, Carlander B, et al. Les potentiels evoques somesthesiques dans la sclerose amyotrophique et la sclerose laterale primaire. Rev Neurol (Paris) 1994;150 $292-8$
29 Zanette G, Tinazzi M, Polo A, et al. Motor neuron disease with pyramidal tract dysfunction involves the cortical gnerators of the early somatosensory evoked potentials to tibial nerve stimulation. Neurology 1996;47:932-8.

30 Marti-Fabregas J, Pujol J. Selective involvement of the pyramidal tract on magnetic resonance imaging in primary lateral sclerosis. Neurology 1990;40:1799-800.

\section{HISTORICAL NOTE}

\author{
Emil Heinrich Du Bois-Reymond \\ (1818-96)
}

This distinguished physiologist is remembered for two dissimilar contributions: he was a founder of electrophysiology of nerve and muscle; and he described his own migraine.

Du Bois-Reymond first discovered that the peripheral passage of a nerve impulse was accompanied by an electrical discharge ${ }^{1}$ the action potential. After Matteucci, whose work he disparaged, he is often regarded as the founder of electrophysiology.

$\mathrm{Du}$ Bois-Reymond was born and studied medicine in Berlin. His father was a watchmaker in the Swiss canton Neuchatel but then moved to Berlin as a civil servant. Emil became a student of Johannes Müller, working with him from 1841 until Müller's death in 1858 . After years of industrious study, he succeeded him to the Chair of Physiology. At that time he was in close contact with Helmholtz, Brücke, and Ludwig, liaisons that culminated in the foundation of a new institute of physiology in Berlin in 1877. Du Bois-Reymond was the chief for 20 years.

He was described in somewhat antiquated terms, a materialistic, and a mechanical physiologist. Du Bois-Reymond invented a refined sensitive nerve galvanometer and a stimulus producing induction coil. $\mathrm{He}$ showed an electric current in muscle (Müskelstrom). It was, he thought, owing to an attempt to preserve a resting (pre-existing) difference of potential between the negative inner and positive outer membranes of the fibre, which caused an electromotive force appearing with injury-an important and new concept:

"The law of muscular current may be shortly expressed as follows: Any point of the natural or artificial longitudinal section of muscle is positive relation to any point of the natural or artificial transverse section ... every particle of a muscle, however minute, ought to produce a current in the same manner as the whole muscle ... As to the nerves ... they are possessed of an electromotive power, which acts according to the same law as muscles."
He confirmed Matteucci's observation that during tetanus, the resting current flowing from an intact to an injured region is decreased and this negative variation was composed of a series of individual variations. This is called the negative variation (negative Schwankung) of Du Bois-Reymond. It now corresponds to variation in the action potential.

$\mathrm{He}$ investigated physiological tetanus ${ }^{2}$ in 1850 and employed in his work the galvanometer. By this means he defined what he called electrotonus, the potential changes produced by an externally applied current; he also experimented with Faradic stimulation.

Du Bois-Reymond is also well known ${ }^{3}$ for his views on the pathogenesis of migraine. Before his time, Robert Whytt had given an exposition of the spasm and relaxation of small blood vessels in migraine $e^{4}$ that foreshadowed the vasospastic theories of Latham. Latham had initiated the vascular hypothesis and explained it as:

"a contraction of the blood vessels of the brain, and so diminished supply of blood, produced by the excited action of the sympathetic; and that the exhaustion of the sympathetic following on this excitement causes the dilatation of the vessels and the headache."

This topic seems remote from the electrophysiological work of Du Bois-Reymond, but his account in 1860 displays a personal stake:

"a Tetanus takes place in the muscular coats of the vessels of the affected half of the head; in other words a Tetanus of the cervical portion of the sympathetic." ${ }^{\prime \prime}$... every three or four weeks I am liable to an attack. I wake with a general feeling of disorder, and a slight pain in the region of the right temple, which, without overstepping the midline, reaches its greatest intensity at midday; towards evening it usually passes off. While at rest the pain is bearable, but it is increased by motion to a high degree of violence ... It responds to each beat of the temporal artery. The latter feels on the affected side, like a hard cord, while the left is in normal condition. The countenance is pale and sunken, the right eye small and reddened ... There may be left behind a slight gastric disorder; frequently, also, the scalp remains tender at one spot the following morning ... For a certain period after the attack I can expose myself with impunity to influences which before would infallibly caused an attack."
This account is of interest showing the remission after an attack; and enophthalmos or miosis, and the red eye, which we would associate with cluster headache. $\mathrm{He}$ also deduced both the vascular component of the headache phase, and the role of the sympathetic nerves in inducing vascular constriction. Later, in 1873, Edward. Liveing in his classic text ${ }^{7}$ accepted that dilatation of the arteries might explain the headache, but like Gowers, Liveing rejected the vascular theory as explanation of the varied content of the aura, its bilaterality in certain patients, the vegetative symptoms throughout the body, and the changes in patterns of attacks. Liveing regarded it as a "nerve storm."

"a form of centrencephalic seizure, the activity of which is projected rostrally upon the cerebral hemispheres, and peripherally via the ramifications of the autonomic nervous system ..."

In explaining his nerve storm theory ${ }^{8}$ Liveing considered migraine along with other "neuroses" to be:

"a primary and often hereditary disposition of the nervous system itself; this consists in a tendency to the irregular accumulation and discharge of the nerve force..."

Time has shown that Liveing's and Gowers' theories are closer to the truth than du Bois Reymond's, though we still do not understand the initial mechanism. jmspearce@freenet.co.uk

$1 \mathrm{Du}$ Bois Reymond E. Vorlaufiger Abriss einer Untersuchung über den sigenannten FroUntersuchung über den sigenannten FroFische. Annl Phys 1843;58:1-30.

$2 \mathrm{Du}$ Bois Reymond E. Untersuchungen über thierische Elektikstät. Vol 2:425-30. Berlin: G Reimer, 1849. Cited in: Clarke E, O'Malley CD. The human brain and spinal cord: a historical study, 2nd ed. San Francisco: Norman, 1996: 192-203.

3 Pearce JMS. Occasional review: historical aspects of migraine. $\mathcal{F}$ Neurol Neurosurg Psychiatry 1986;49:1097-103.

4 Whytt R. Observations on the nature, causes and cure of those disorders commonly called nervous, hypochondriac, or histeric. Edinburgh: Becket, Pond, and Balfour, 1764.

5 Latham PW. The pathology of sick-headache. BMF 1893;i:113-4.

6 Du Bois-Reymond E. Leipzig: Arch Anat Physiol 1860;461-8.

7 Liveing E. On megrim, sick headache, and some allied disorders: a contribution to the pathology of nerve storms. London: Churchill, 1873.

8 Pearce JMS. Edward Liveing's theory of nerve storms in migraine. In: FC Rose, ed. A short history of neurology. Oxford: Butterworth, 1999: 192-203. 